\title{
Les femmes dans la recherche : les actions de la SFP
}

Comme d'autres sciences (mathématiques, sciences de l'information...), la physique souffre actuellement d'une désaffection des jeunes, et en particulier des jeunes filles, alors que leur présence dans les laboratoires est primordiale et bénéfique à beaucoup de points de vue. De nombreuses associations ${ }^{(a)}$ et institutions officielles $^{(b)}$ se penchent sur le problème et proposent des solutions pour améliorer la situation. La Société Française de Physique se doit de participer à cet effort général pour améliorer la place des femmes dans la recherche et augmenter leur visibilité dans les métiers de la physique. C'est à travers ces modèles que la recherche en physique attirera des jeunes femmes.

À la suite des réunions de son bureau et de son conseil (1 ${ }^{\mathrm{er}}$ et 18 mars 2016), la Société Française de Physique, sur recommandation de sa commission Femmes et Physique, a décidé d'appliquer la loi pour les jurys des prix $\mathbf{( 4 0} \%$ de femmes au minimum, en essayant de viser 50\%). Les présidents des différents prix de la SFP devront, au travers des processus de nomination, veiller à atteindre ces objectifs sur une période de cinq ans.

Les conférenciers pléniers ainsi que les comités scientifiques et d'organisation des congrès devront désormais comporter un minimum de $\mathbf{2 0} \%$ de femmes, et viser si possible une proportion de 40 à $50 \%$.

La commission Femmes et Physique de la SFP œuvre pour une meilleure représentation des femmes en physique. Pour cela, entre autres actions, elle a développé et met notamment à disposition :

- une base de données de conférencières et d'expertes (sélectionnées sur recommandation) pour les scolaires, le grand public et les physiciens ;

- un mentorat pour jeunes chercheuses, qui consiste à trouver une femme d'expérience comme interlocutrice pour une femme plus jeune qui en fait la demande ; ce qui peut bien sûr être une première étape avant d'orienter cette dernière vers les services spécialisés de son organisme ;

- un groupe facebook «Femmes et Physique »: Femmes et Physique Public Group | Facebook.

Pour toute suggestion ou demande, écrire à sfp-femmes@sfpnet.fr. La commission Femmes et Physique fera régulièrement un bilan de toutes ces actions.

Nous invitons aussi les membres de la SFP à signer le manifeste Pour les Femmes et la Science de L'Oréal -UNESCO, avec lequel nous sommes vraiment en phase et dont les signataires s'engagent à :

1/ Susciter des vocations scientifiques chez les jeunes filles.

2/ Faire tomber les barrières qui empêchent les femmes de science de poursuivre une carrière à long terme.

3/ Favoriser l'accès des femmes aux postes scientifiques de haut niveau et aux postes décisionnels.

4/ Célébrer auprès du grand public la contribution des femmes scientifiques aux progrès de la science et de la société.

5/ Garantir la parité dans les instances et manifestations scientifiques (colloques, commissions et conseils d'administration) en termes de participation et de leadership.

6/ Favoriser le mentorat et l'accès aux réseaux pour les jeunes scientifiques, afin de leur permettre de planifier et de développer leurs carrières à la hauteur de leurs espérances.

Par ailleurs, début 2012, les trois associations Femmes \& mathématiques, Femmes \& Sciences et Femmes Ingénieurs, s'étaient unies pour interpeller les candidat-e-s à la Présidence de la République sur le thème «femmes, sciences et éducation ». Ces associations avaient choisi d'aborder le sujet en élaborant vingt propositions pragmatiques à l'efficacité reconnue, au coût marginal et nécessitant surtout une volonté politique, pour un objectif d'intérêt général.

Les vingt propositions étaient classées en quatre thèmes :

1/ Déconstruire les idées reçues de la société concernant les femmes et les sciences.

2/ Encourager l'orientation des jeunes, et plus particulièrement des jeunes filles, vers les filières scientifiques et techniques.

(a) Femmes \& Sciences, Femmes \& Mathématiques, Femmes Ingénieurs...

(b) Ministère de I'Enseignement supérieur et de la Recherche, Mission pour la place des femmes au CNRS, CEA, Conférence

Permanente des chargé-e-s de mission Égalité-Diversité des établissements

d'enseignement supérieur et de recherche (CPED)...

3/ Repenser la place des sciences et de l'ingénierie dans l'éducation.

4/ Faciliter la carrière des femmes scientifiques, techniciennes et ingénieures.

La SFP s'associe à la réflexion pour faire évoluer ces propositions pour les élections à la Présidence de la République de 2017. Afin de vous permettre de vous exprimer sur l'évolution de la situation et les points éventuels à ajouter, un questionnaire a été mis en ligne avec le lien :

https://femmesscing.evalandgo.com/s/?id=JTk1ayU5MmolOTclQjl=\&a=JTk1aSU5MmklOUMIQjl=

Plus les réponses seront nombreuses dans cette première phase de réflexion, plus nous serons représentatif.ve.s. Merci d'être nombreux.ses à répondre à ce questionnaire !

Michel Spiro

Président de la Société Française de Physique
Véronique Pierron-Bohnes

Présidente de la commission Femmes et Physique de la SFP 